\title{
Voltage-driven annihilation and creation of magnetic vortices in Ni discs
}

M. Ghidini ${ }^{1,2,3, *}$, R. Mansell ${ }^{4, \#}$, R. Pellicelli ${ }^{1,5}$, D. Pesquera ${ }^{3}$, B. Nair ${ }^{3}$, X. Moya ${ }^{3}$,

S. Farokhipoor ${ }^{3}$, F. Maccherozzi ${ }^{2}$, C. H. W. Barnes ${ }^{4}$, R. P. Cowburn ${ }^{4}$, S. S. Dhesi ${ }^{2}$ and N. D. Mathur ${ }^{3, \dagger}$

${ }^{1}$ Department of Mathematics, Physics and Computer Science, University of Parma, 43124 Parma, Italy.

${ }^{2}$ Diamond Light Source, Chilton, Didcot, Oxfordshire, OX11 0DE, UK.

${ }^{3}$ Department of Materials Science, University of Cambridge, Cambridge, CB3 OFS, UK.

${ }^{4}$ Cavendish Laboratory, University of Cambridge, CB3 OHE, UK.

${ }^{5}$ Istituto d'Istruzione Superiore A. Zanelli, 42123 Reggio Emilia, Italy.

\# Now at NanoSpin, Department of Applied Physics, Aalto University, FI-00076 Aalto, Finland

*massimo.ghidini@unipr.it

$\dagger$ †dm12@cam.ac.uk

Using photoemission electron microscopy (PEEM) to image ferromagnetism in polycrystalline $\mathrm{Ni}$ disks, and ferroelectricity in their single-crystal $\mathrm{BaTiO}_{3}$ substrates, we find that voltage-driven $90^{\circ}$ ferroelectric domain switching serves to reversibly annihilate each magnetic vortex via uniaxial compressive strain, and that the orientation of the resulting bi-domain reveals the chirality of the annihilated vortex. (Micromagnetic simulations reveal that only $60 \%$ of this strain was required for annihilation.) Voltage control of magnetic vortices is novel, and should be energetically favourable with respect to the use of a magnetic field or an electrical current. In future, stray field from bi-domains could be exploited to read vortex chirality. Given that core polarity can already be read via stray field, our work represents a step towards four-state low-power memory applications.

The technological goal of developing low-power electric-write magnetic-read memories is driving sustained research effort into the electrical control of magnetism [1-5]. This converse magnetoelectric effect has now been demonstrated in single-phase multiferroic 
materials [6-10] as well as composite multiferroic materials [11-29]. The latter perform well at room temperature because they comprise room-temperature ferroelectrics and room-temperature ferromagnets, coupled via strain [21-25], charge [26] or exchange bias [27-29]. These magnetoelectric coupling mechanisms permit electric fields to switch inplane magnetic domains [17], modify a large in-plane magnetization [14,30], switch a large magnetization from out-of-plane to in-plane [21], reorient magnetic easy axes $[15,16]$, modulate coercivity [13], and control magnetic domain wall motion [31].

Separately, magnetic vortices have been demonstrated in discs of permalloy [32,33], iron [34] and nickel [35]. Vortex geometry is constrained by details of the host material, such that discs with vortices cannot be miniaturized down to the sub-10 nm dimensions associated with state-of-the-art magnetic tunnel junctions [36]. However, vortices have nevertheless attracted much interest in the context of magnetic data storage because they possess four well-defined stable states [37,38]. Specifically, a $\sim 10 \mathrm{~nm}$-wide vortex core [34] can be magnetized up or down to yield two states of polarity, while the curl of the surrounding in-plane magnetization can take either sign to yield two states of chirality. The ability to independently switch core polarity $[39,40]$ and vortex chirality [41,42] raises hopes of four-state memory [43], and to reduce power consumption it would be attractive to drive such changes with a voltage (nondeterministic voltage-driven switching of vortex chirality was demonstrated in ref. [44]).

Here we exploit strain-mediated magnetoelectric coupling from a single-crystal substrate of $\mathrm{BaTiO}_{3}$ (BTO) in order to electrically annihilate/create magnetic vortices in polycrystalline Ni discs of thickness $25 \mathrm{~nm}$ and diameter $1 \mu \mathrm{m}$. By imaging both the ferromagnetic domains and the ferroelectric domains, we found that pre-existing vortices were annihilated when a voltage drove $a \rightarrow c$ ferroelectric domain switching in the underlying substrate, and that subsequent $c \rightarrow a$ switching reversed this annihilation. Micromagnetic simulations reproduce the observed magnetoelectric effects with reasonable fidelity, and suggest that our vortices were annihilated by a change of strain that is just $60 \%$ of the uniaxial $10^{-2}$ strain associated with $a \rightarrow c$ switching. Importantly, vortex chirality was found to uniquely determine the orientation of the bi-domains that resulted from vortex annihilation. This immediately suggests that one could identify the chirality of vortices in memory devices by converting them to bi-domains, and reading the lateral stray field. 


\section{Experimental methods}

A $200 \mathrm{~nm}$-thick Pt electrode was sputter-deposited on the back of a $0.5 \mathrm{~mm}$-thick BTO substrate that adopted the pseudo-cubic (001) orientation. After ultrasonic cleaning with first acetone, then isopropyl alcohol and last deionised water, we used thermal evaporation to deposit first Ta and then Pt to form a $2 \mathrm{~nm}$-thick top electrode of $\mathrm{Ta}(0.5 \mathrm{~nm}) / \mathrm{Pt}(1.5 \mathrm{~nm})$. We subsequently used electron-beam-assisted evaporation with a base pressure of $3 \times 10^{-10}$ mbar to grow $25 \mathrm{~nm}$ of $\mathrm{Ni}$, capped with $3 \mathrm{~nm}$ of $\mathrm{Cu}$, through a lift-off mask defined by electron-beam lithography in a double layer of PMMA resist. This resulted in $10 \times 10$ arrays of $1 \mu \mathrm{m}$-diameter Ni discs, with nearest-neighbour centre-to-centre separations of $2 \mu \mathrm{m}$ [Fig. 1(a)]. Ni discs of this size are known to display vortices [35], whereas thinner Ni discs form single-domain states $[35,45]$.

Raw PEEM images $(1024 \times 1024$ pixels $)$ were obtained in zero applied magnetic field on beamline I06 at Diamond Light Source, where we used an Elmitec SPELEEM-III microscope to map secondary-electron emission arising from circularly polarized $\mathrm{x}$-rays that were incident on the sample surface at a grazing angle of $16^{\circ}$. The probe depth of $\sim 7 \mathrm{~nm}$ was sufficient to sample the $\mathrm{Ni}$ discs through their $3 \mathrm{~nm}$-thick $\mathrm{Cu}$ caps, and to sample the surrounding BTO surface through the $2 \mathrm{~nm}$-thick top electrode of $\mathrm{Pt} / \mathrm{Ta}$. The lateral resolution was typically $\sim 50 \mathrm{~nm}$. A $300 \mathrm{~V}$ power supply was connected to the top and bottom electrodes via feedthroughs in the sample holder.

Raw XMCD-PEEM images were acquired during $1 \mathrm{~s}$ exposure times with right $(\mathrm{R})$ and left $(\mathrm{L})$ circularly polarized light, both on the $\mathrm{Ni} L_{3}$ resonance at $851 \mathrm{eV}$, and off this resonance at $842 \mathrm{eV}$. The pixels in a raw XMCD-PEEM image describe the XMCD asymmetry $\left(I^{\mathrm{R}}-I^{\mathrm{L}}\right) /\left(I^{\mathrm{R}}+I^{\mathrm{L}}\right)$, which represents the projection of the local surface magnetization on the incident-beam direction. Here, $I^{\mathrm{R} / \mathrm{L}}=\left(I_{\mathrm{on}}^{\mathrm{R} / \mathrm{L}}-I_{\text {off }}^{\mathrm{R} / \mathrm{L}}\right) / I_{\mathrm{off}}^{\mathrm{R} / \mathrm{L}}$ denotes the relative intensity for secondary electron emission due to x-ray absorption on $\left(I_{\mathrm{on}}^{\mathrm{R} / \mathrm{L}}\right)$ and off $\left(I_{\mathrm{off}}^{\mathrm{R} / \mathrm{L}}\right)$ the Ni $L_{3}$ resonance (the comparison between intensities obtained on and off resonance avoids the influence of any inhomogeneous illumination). The pixels in a raw XAS-PEEM image describe the $x$-ray absorption (XAS) intensity $I_{\mathrm{on}}^{\mathrm{R}}+I_{\mathrm{on}}^{\mathrm{L}}$, which represents topographical/chemical contrast. 
Raw XLD-PEEM images were acquired during $1 \mathrm{~s}$ exposure times with vertically (V) and horizontally $(\mathrm{H})$ polarized light, both on the $\mathrm{Ti} L_{3}$ resonance at $457 \mathrm{eV}$, and off this resonance at $446 \mathrm{eV}$. The pixels in a raw XLD-PEEM image describe the XLD asymmetry $\left(I^{\mathrm{V}}-I^{\mathrm{H}}\right) /\left(I^{\mathrm{V}}+I^{\mathrm{H}}\right)$, which represents the projection of the local surface polarization on the incident-beam direction. Here, $I^{\mathrm{V} / \mathrm{H}}=\left(I_{\mathrm{on}}^{\mathrm{V} / \mathrm{H}}-I_{\mathrm{off}}^{\mathrm{V} / \mathrm{H}}\right) / I_{\text {off }}^{\mathrm{V} / \mathrm{H}}$ denotes the relative intensity for secondary electron emission due to $\mathrm{x}$-ray absorption on $\left(I_{\mathrm{on}}^{\mathrm{V} / \mathrm{H}}\right)$ and off $\left(I_{\mathrm{off}}^{\mathrm{V} / \mathrm{H}}\right)$ the $\mathrm{Ti} L_{3}$ resonance.

All raw PEEM images were averaged for display or the subsequent construction of magnetic vector maps. We averaged 100 raw XMCD-PEEM images to obtain XMCD-PEEM images of ferromagnetic domains. We averaged 100 raw XLD-PEEM images to obtain XLD-PEEM images of ferroelectric domains. We averaged 100 raw XAS-PEEM images to obtain XAS-PEEM images of topographical/chemical contrast. Magnetic vector maps were obtained by combining two such XMCD-PEEM images for orthogonal sample orientations, after correcting for drift and distortion via an affine transformation that was based on the averaged XAS-PEEM image for each sample orientation.

Micromagnetic simulations performed using MuMax3 [46] involved discretizing a Ni disc of the dimensions employed experimentally into $5 \times 5 \times 2.5 \mathrm{~nm}^{3}$ cells, assuming an exchange stiffness of $0.82 \times 10^{-11} \mathrm{~J} \mathrm{~m}^{-1}$ [47] and a saturation magnetization of $434 \mathrm{kA} \mathrm{m}^{-1}$ [21]. The uniaxial $y$-axis strain $\varepsilon_{\mathrm{y}}$ from the substrate was assumed to introduce magnetic stress anisotropy energy density $-K \cos ^{2} \theta$, where $\theta$ is the angle between the in-plane magnetization and the uniaxial strain, $K=\frac{3}{2} E \varepsilon_{\mathrm{y}} \lambda$, Young's modulus $E=133 \mathrm{GPa}$, and $\mathrm{Ni}$ magnetostriction $\lambda=-32.9 \times 10^{-6}[21]$. Stable equilibrium configurations were identified by dynamically relaxing the magnetization, and these configurations were confirmed via minimizations that exploited the conjugate gradient method [46]. In both of these methods, MuMax3 internally suppresses the precessional term in the Landau-Lifschitz-Gilbert equation, and the user-defined damping constant is ignored. Images and polar plots describe the average magnetization of the upper two simulation cells, whose $5 \mathrm{~nm}$ thickness is similar to the $\sim 7 \mathrm{~nm}$ PEEM probe depth. Image quality was improved by plotting four pixels for each of the 32428 pairs of double-height cells. 


\section{Experimental results}

The Ni discs can be seen in the XAS-PEEM image shown in Fig. 1(b), where the field of view represents a diameter of $15 \mu \mathrm{m}$. On cycling a voltage that was applied across the entire BTO substrate and varied in discrete intervals, we identified notable changes in XMCD-PEEM images for $0 \mathrm{~V} \rightarrow 150 \mathrm{~V} \rightarrow-300 \mathrm{~V} \rightarrow 0 \mathrm{~V}$. At these voltages, we obtained XMCD-PEEM images of disc 1 for one in-plane projection of the incident X-ray beam [Fig. 2(a-d)] and its orthogonal counterpart [Fig. 2(e-h)]. For each voltage, the two XMCD-PEEM images were used to identify the near-surface magnetization direction $\alpha$, which we present on magnetic vector maps [Fig. 2(i-l)] and polar histograms [Fig. 2(m-p)]. Vector maps can be difficult to infer by visual inspection from the two constituent XMCD-PEEM images, as the contrast in these images is shown on a colour scale with poor resolution.

The vortex state in the virgin disc was anisotropic, given that polar-plot lobes were oriented somewhat along $x$. For negative-magnetostriction $\mathrm{Ni}$, this suggests the possibility of tensile strain along $y$. Polycrystalline films can indeed experience tensile growth strain from BTO substrates along the long $c$ axis of $a$ domains [17,45], possibly due to some degree of epitaxy. However, this indirect evidence for an $a$ domain polarized along $y$ is not compelling, as disc magnetization is not uniform, and the observed anisotropy could also arise from substrate-independent growth strain and any disc eccentricity. Instead, we will identify the ferroelectric domain structure via XLD-PEEM, but first we will discuss the effect of the voltage cycle on disc 1.

The anisotropic vortex in the virgin disc was annihilated when $150 \mathrm{~V}$ stabilized a bi-domain whose absolute domain orientations follow from the chirality of the annihilated vortex. At $-300 \mathrm{~V}$, the two magnetic domains adopted better antiparallel alignment, and a concomitantly straighter domain wall. Given that these two large bipolar voltages resulted in similar bi-domains, we may infer that they caused the disc to experience similar strains from oppositely polarized ferroelectric $c$ domains. It is therefore natural to anticipate that vortex annihilation is driven by $a \rightarrow c$ ferroelectric domain switching, as we will show below via Fig. 3. A vortex was recovered on returning to $0 \mathrm{~V}$, suggesting that $c \rightarrow a$ switching took place underneath. This recovered vortex possessed the same chirality as the starting vortex, given our observation that chirality information was stored in the absolute domain orientation of the 
bi-domain. The polar plots show that the recovered vortex possesses greater anisotropy than the starting vortex, evidencing irreversible strain.

Fig. 3(a) is a composite image that describes the virgin state of the sample in our $15 \mu \mathrm{m}$-diameter field of view. The vector map of disc 1 is accompanied by a vector map of disc 2, revealing another anisotropic vortex with opposite chirality. The XMCD-PEEM image representing the other discs reveals anisotropic vortices with different chiralities. The XLD-PEEM image representing the BTO between discs does not contain any domain walls, implying that the field of view shows a single ferroelectric domain. Below we will identify this ferroelectric domain to be $a$ type.

Fig. 3(b) corresponds to Fig. 3(a) after applying the $150 \mathrm{~V}$. The vector map of disc 1 is accompanied by a vector map of disc 2 , revealing another bi-domain with the same easy axis. However, the two domain magnetizations were each reversed due to the opposite chirality of the virgin state. The XMCD-PEEM image representing the other discs reveals bi-domains with the same easy axis as discs 1 and 2, while the absolute directions of magnetization likewise depend on virgin chirality. The XLD-PEEM image representing the BTO between discs reveals the presence of two outer ferroelectric domains that were associated with all of the observed discs, and a middle domain that was continuously annihilated over the course of several minutes (not shown). We attribute this lack of stability to an elastic energy cost associated with the unexpected [48] meeting of long $c$ axes and short $a$ axes at the two observed walls. We may consequently deduce that the as-marked $c-a-c$ configuration [Fig. 3(b)] arose from $a \rightarrow c$ switching under the discs, which thus experienced compressive strain along $y$ to yield the observed easy axis along $y$. The complementary $a-c-a$ configuration can be ruled out because it would imply that the discs experienced $c \rightarrow a$ switching with $a$ domains polarized along $y$, i.e. tensile strain along $y$ and thus an easy axis along $x$.

After completing the cycle shown in Fig. 2, vector maps of disc 2 (not shown) confirm that the virgin vortex chirality had also been restored in this disc, consistent with the reversed bi-domain that we observed at finite voltage [Fig. 3(b)]. The corresponding XLD-PEEM images at $-300 \mathrm{~V}$ and $0 \mathrm{~V}$ were not measured. However, XMCD-PEEM images (not shown) confirmed that the bi-domain was stable at $0 \mathrm{~V}$. 


\section{Modelling}

For an unstrained Ni disc of the dimensions employed here, the lowest energy state would be an isotropic vortex [Fig. 4(a-c)], as expected [35], and the core would be $30 \mathrm{~nm}$ wide [Fig. 4(b)]. Introducing the inferred tensile growth strain along $y$ resulted in an anisotropic vortex [Fig. 5(a,e,i)], where we have set $\varepsilon_{y}=2 \times 10^{-3}$ to best match the anisotropic vortex state of discs 1 and 2 in the virgin sample [Fig. 3(a)]. The resulting value of $K=\frac{3}{2} E \varepsilon_{\mathrm{y}} \lambda=-10 \mathrm{~kJ} \mathrm{~m}^{-3}$ has the expected order of magnitude for Ni on BTO [21].

Replacing the $y$-axis tensile strain arising from growth with a $y$-axis compressive strain due to the $a \rightarrow c$ switching observed at $150 \mathrm{~V}$ [Fig. 3(b)] resulted in a bi-domain [Fig. 5(b,f,j)], where we have set $\varepsilon_{y}=-4 \times 10^{-3}\left(K=20 \mathrm{~kJ} \mathrm{~m}^{-3}\right)$ to best match the bi-domain in disc 1 at $150 \mathrm{~V}$ [Fig. 2(j,n)]. The simulated bi-domain at $150 \mathrm{~V}$ is $3 \%$ more energetically expensive than a vortex state that we modelled at the same strain (not shown). However, an unexplored energy barrier will determine the related issues of stability and interconversion, and experimentally the vortices and bi-domains were found to persist over many hours at a given voltage while collecting XMCD-PEEM images. The domain wall in the simulated bi-domain at $150 \mathrm{~V}$ is seen to bend and widen at the top, explaining the slightly imperfect lobe alignment in the corresponding polar plot. This finding is reminiscent of our observation that disc 1 displays a wavy domain wall at $150 \mathrm{~V}$, and misaligned polar-plot lobes.

Increasing the $y$-axis compressive strain to model the subsequent application of a larger voltage with opposite sign resulted in an asymmetric bi-domain [Fig. 5(c,g,k)], where setting $\varepsilon_{y}=-5 \times 10^{-3}\left(K=25 \mathrm{~kJ} \mathrm{~m}^{-3}\right)$ achieved good agreement with the asymmetric bi-domain in disc 1 at $-300 \mathrm{~V}$ [Fig. 2(k,o)]. The agreement is better at $-300 \mathrm{~V}$ than $150 \mathrm{~V}$, implying the possibility that switching reduced some uncontrolled long-range strain from unobserved ferroelectric domains. On reaching $300 \mathrm{~V}$, the growth of one domain at the expense of the other can be understood as a step towards the single-domain state expected at sufficiently high strain. Switching the $y$-axis strain to tensile in order to model the final $c \rightarrow a$ switching step resulted in an anisotropic vortex [Fig. 5(d,h,l)], where we have exceeded the growth strain of $\varepsilon_{y}=2 \times 10^{-3}$ by setting $\varepsilon_{y}=5 \times 10^{-3}\left(K=-25 \mathrm{~kJ} \mathrm{~m}^{-3}\right)$ to best match the more anisotropic vortex that arose in disc 1 after returning to $0 \mathrm{~V}$ [Fig. 2(1)]. 


\section{Discussion}

Let us now consider the magnitudes of the strains that we have used to model vortex annihilation in disc 1 . By overcoming the tensile growth strain of $2 \times 10^{-3}$ with a compressive strain of $-4 \times 10^{-3}$, we may infer that $a \rightarrow c$ switching resulted in a compressive strain of $6 \times 10^{-3}$. This represents $60 \%$ of the uniaxial $10^{-2}$ strain that one would expect from a $90^{\circ}$ rotation of the BTO unit cell, implying the likelihood of imperfect strain transmission. The increase of strain that we identified with the magnetic changes observed from $150 \mathrm{~V}$ to $-300 \mathrm{~V}$ is likely to be associated with the concomitant ferroelectric domain reversal, as the increase of strain associated with the continuous piezoelectric effect [21] is two orders of magnitude too small to explain our calculated strain increase.

In summary, we have used XMCD-PEEM imaging and XLD-PEEM imaging to demonstrate that voltage-driven $90^{\circ}$ ferroelectric domain switching in a BTO substrate can reversibly annihilate magnetic vortices in $1 \mu \mathrm{m}$-diameter Ni discs, and that the chirality of the annihilated vortex determines the orientation of the resulting bi-domain. We subsequently used micromagnetic modelling to suggest that vortex annihilation was achieved with $60 \%$ of the strain that one might expect for the ferroelectric domain switching, which demonstrates scope for optimization. The reversible magnetoelectric annihilation that we have demonstrated should in future permit vortex chirality to be read via lateral stray field, just as the polarity of a vortex can be read via perpendicular stray field.

Ultimately, it would be interesting to read all four stable vortex states [43] via stray field, using the smallest discs of a given material to show vortices [35]. To exploit four-state vortex memory in low-power electric-write magnetic-read memory devices would require two key improvements. First, it would be important to overcome the key challenge of electrically switching chirality and polarity, possibly via inhomgenous/dynamic electrically driven strain. Second, it would be important to define individually addressable ferroelectric elements that overcome clamping from a fixed substrate, possibly via pillars with a high aspect ratio. These improvements could also be relevant for magnetoelectric control of magnetic skyrmions.

\section{Acknowledgments}

We acknowledge Diamond Light Source for time on I06 under proposal SI11843. X.M. is grateful for support from the Royal Society. B.N. is grateful for the support from Gates 
Cambridge, the Winton Programme for the Physics of Sustainability and Trinity College (Cambridge). D.P. acknowledges Agència de Gestió d'Ajuts Universitaris i de Recerca (AGAUR) from the Catalan government for Beatriu de Pinós postdoctoral fellowship (2014 BP-A 00079). 
(a)

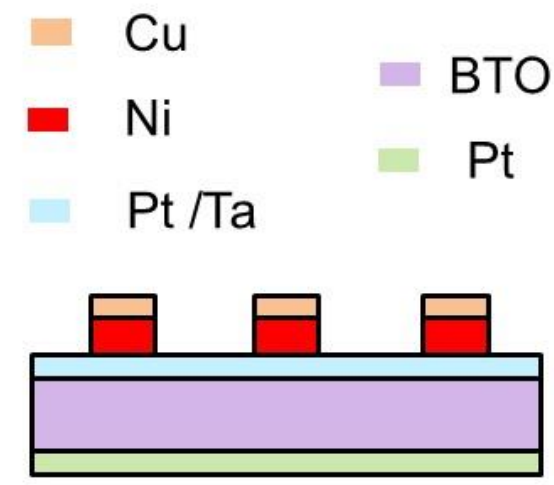

(b)

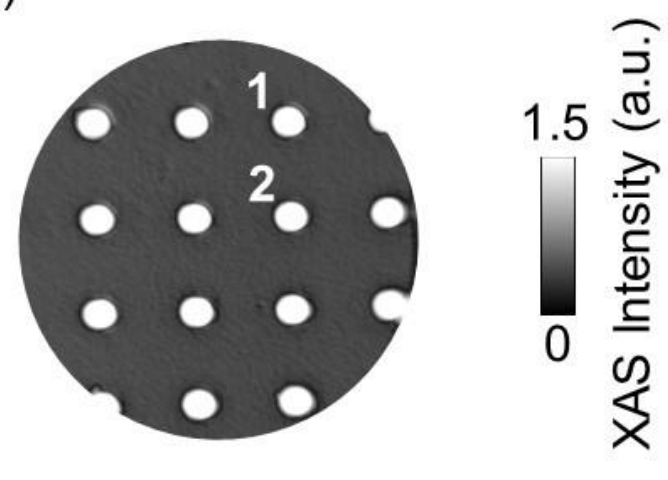

Fig. 1. Sample schematic and area of interest. (a) Cross-sectional schematic showing three of many $\mathrm{Cu}$-capped $\mathrm{Ni}$ discs on an electroded BTO substrate in the pseudo-cubic (001) orientation. (b) XAS-PEEM image, obtained in the as-shown $15 \mu \mathrm{m}$-diameter field of view. Disc diameter $1 \mu \mathrm{m}$, Ni thickness $25 \mathrm{~nm}$. Labels identify discs 1 and 2 . 


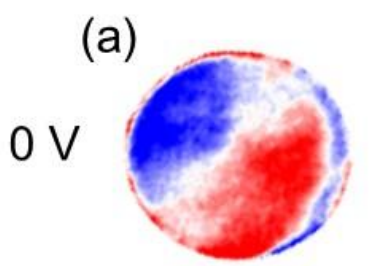

(e)

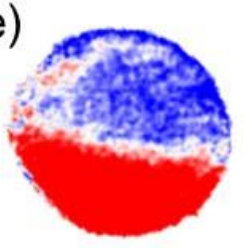

(b)

$150 \mathrm{~V}$
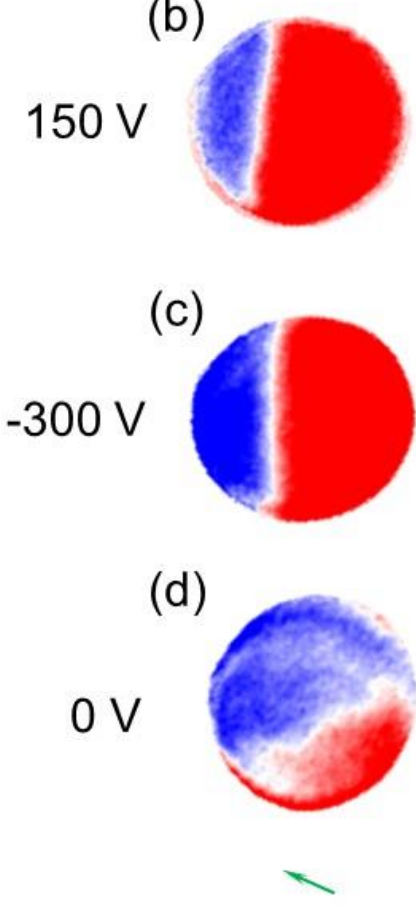

(f)

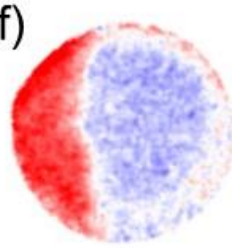

(g)

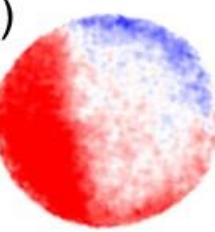

(h)

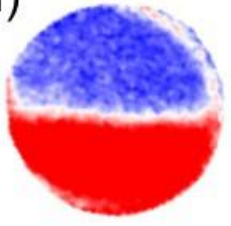

1 (i)

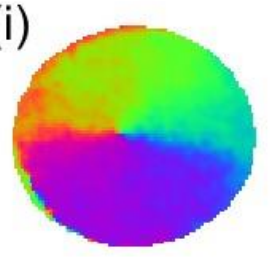

(j)

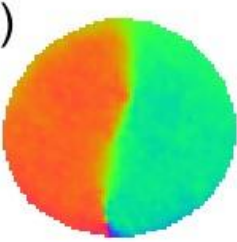

(k)

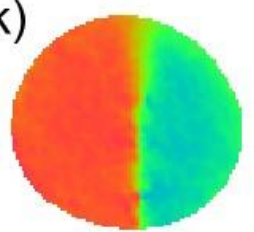

(I)
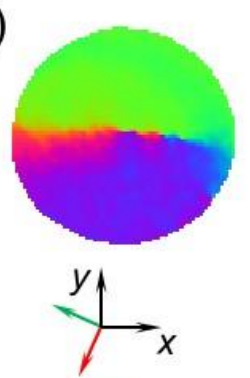

0.1

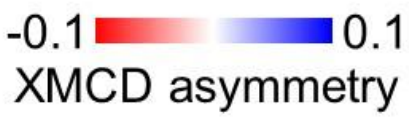

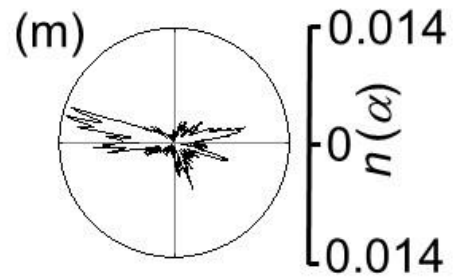

(n)

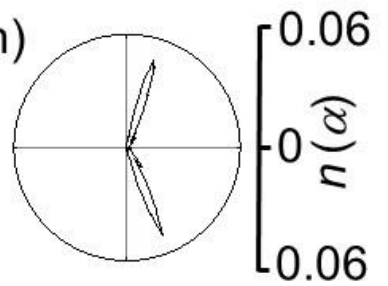

(o)

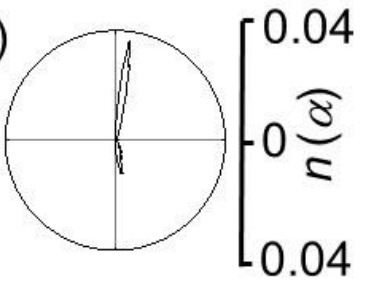

(p)

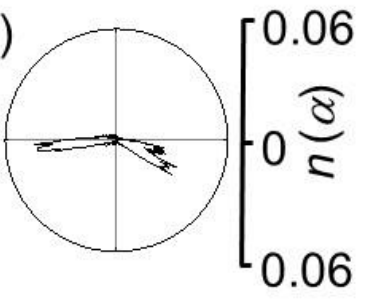

Fig. 2. Magnetic images of disc 1 under voltage control. (a-h) XMCD-PEEM images obtained while applying the specified voltages across the BTO substrate, with the in-plane projection of the grazing-incidence X-ray beam parallel to (a-d) the red arrow and (e-h) the green arrow. Pairwise image combination yields near-surface magnetization direction $\alpha$, which we present on (i-l) magnetic vector maps and (m-p) polar histograms. Histograms show the fraction of pixels $n$ with in-plane magnetization direction $\alpha$ after binning at $1^{\circ}$. Data obtained in a $10 \mu \mathrm{m}$-diameter field of view. Disc diameter $1 \mu \mathrm{m}$. Vector maps in $(\mathrm{j}, \mathrm{k})$ also appear in Fig. 3(a,b). 
(a)

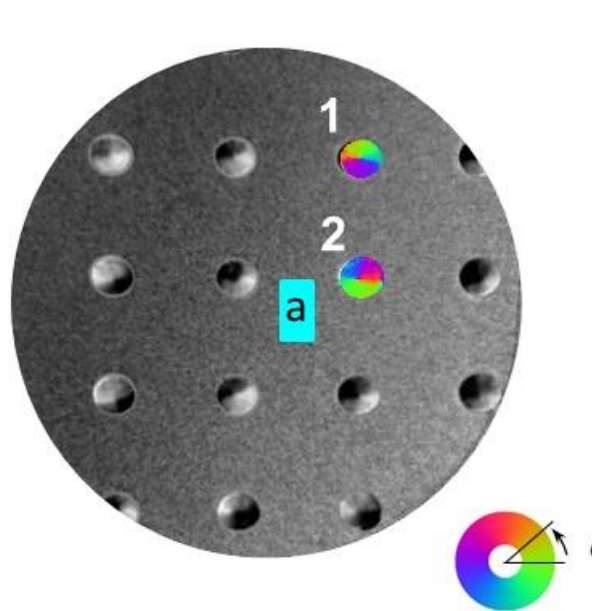

(b)

$150 \mathrm{~V}$

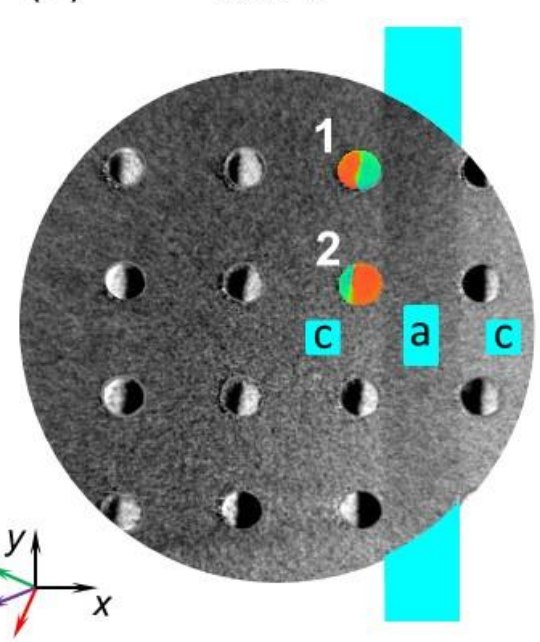

XMCD asymmetry

$-0.1 \square 0.1$

XLD asymmetry

Fig. 3. Voltage-driven switching of ferroelectric domains and disc magnetization. Composite images at (a) $0 \mathrm{~V}$ and (b) $150 \mathrm{~V}$, which each show magnetic vector maps of the near-surface magnetization direction $\alpha$ for discs 1 and 2, an XMCD-PEEM image of other Ni discs, and an XLD-PEEM image of the surrounding ferroelectric domains. BTO unit cell schematics overlie $a$ and $c$ domains. Blue bars in (b) help identify the surviving $a$ domain. The XMCD-PEEM and XLD-PEEM images were obtained in the as-shown $15 \mu \mathrm{m}$-diameter field of view that we used for Fig. 1(b), with the in-plane projection of the grazing-incidence X-ray beam parallel to the purple arrow. The vector maps were constructed from XMCD-PEEM images that were obtained in a $10 \mu \mathrm{m}$-diameter field of view, with the in-plane projections of the grazing-incidence X-ray beam parallel to the red and green arrows. Disc diameter $1 \mu \mathrm{m}$. The two vector maps for disc 1 also appear in Fig. 2(j,k). 
(a)

No strain

$\varepsilon_{y}=0$ (b)

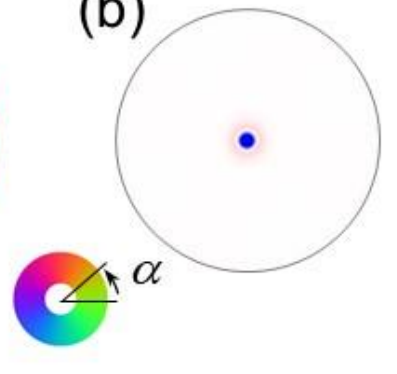

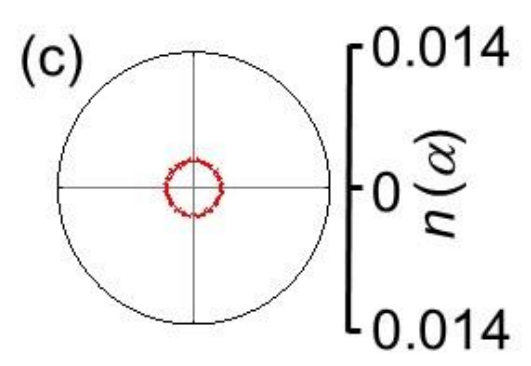

Fig. 4. Micromagnetic modelling of a Ni disc with no growth strain. We present the near-surface magnetization via (a) a map of in-plane magnetization direction $\alpha$, (b) a map of out-of-plane magnetization $M_{z}$, and (c) a polar histogram of $\alpha$. Histogram shows the fraction of pixels $n$ with in-plane magnetization direction $\alpha$ after binning at $1^{\circ}$. The magnitude of the local magnetization is denoted $M$. Disc diameter $1 \mu \mathrm{m}$. 
(a)

$$
\begin{gathered}
\varepsilon_{y}=2 \times 10^{-3} \\
0 \mathrm{~V}
\end{gathered}
$$

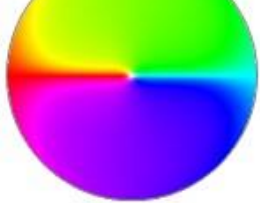

$$
\begin{gathered}
\varepsilon_{y}=-4 \times 10^{-3} \\
150 \mathrm{~V}
\end{gathered}
$$

(b)

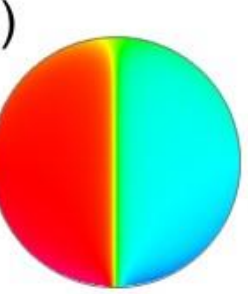

(c)

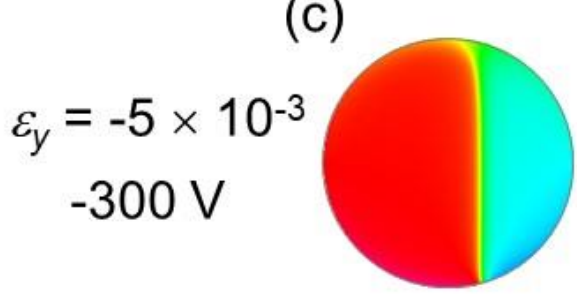

(d)

$\varepsilon_{y}=5 \times 10^{-3}$ $0 \mathrm{~V}$ (e)

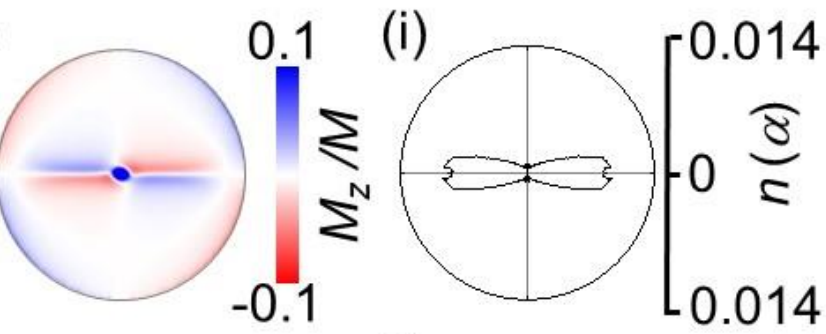

(f)

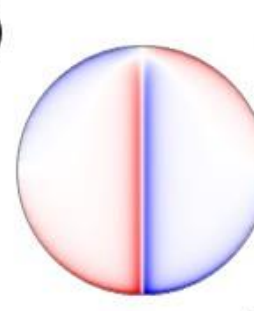

(g)

0.1

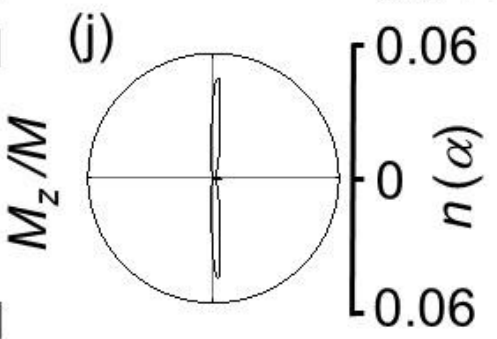

$-0.1$

0.1

(k)

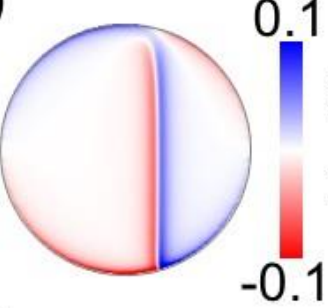

(h)

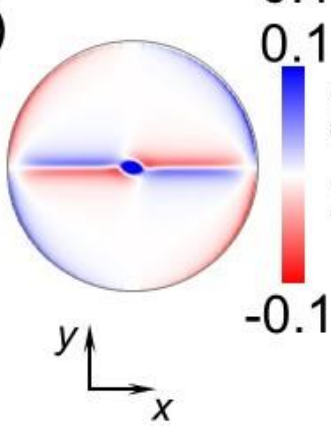

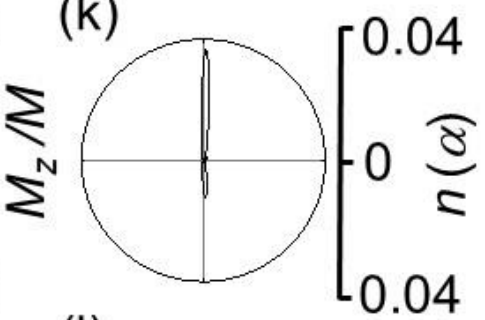

(I)

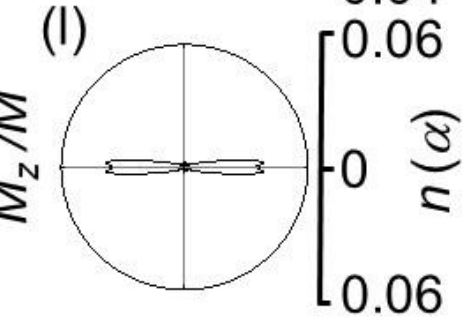

Fig. 5. Micromagnetic modelling of Ni discs under voltage control. For the uniaxial strains $\varepsilon_{y}$ that we identify for our experimentally applied voltages, we present the near-surface magnetization via (a-d) maps of in-plane magnetization direction $\alpha$, (e-h) maps of out-of-plane magnetization $M_{z}$, and (i-l) polar histograms of $\alpha$. Histograms show the fraction of pixels $n$ with in-plane magnetization direction $\alpha$ after binning at $1^{\circ}$. The magnitude of the local magnetization is denoted $M$. Disc diameter $1 \mu \mathrm{m}$. 


\section{References}

[1] M. Fiebig, J. Phys. D, 38 (2005) R123.

[2] W. Eerenstein, N. D. Mathur and J. F. Scott, Nature 442 (2006) 759.

[3] R. Ramesh and N. A. Spaldin, Nature Materials 6 (2007) 21.

[4] S.-W. Cheong and M. Mostvoy, Nature Materials 6 (2007) 13.

[5] C.-W. Nan, M. I. Bichurin, S. Dong, D. Viehland and G. Srinivasan, J. Appl. Phys. 103 (2008) 031101.

[6] D. Lebeugle, D. Colson, A. Forget, M. Viret, A. M. Bataille and A. Gukasov, Phys. Rev. Lett. 100 (2008) 227602.

[7] Y. J. Choi, C. L. Zhang, N. Lee and S.-W. Cheong, Phys. Rev. Lett. 105 (2010) 097201.

[8] Y. Tokunaga, Y. Taguchi, T. H. Arima and Y. Tokura, Nature Physics 8 (2012) 838.

[9] S. H. Chun, Y. S. Chai, B.-G. Jeon, H. J. Kim, Y. S. Oh, I. Kim, H. Kim, B. J. Jeon, S. Y. Haam, J.-Y. Park, S. H. Lee, J.-H. Chung, J.-H. Park and K. H. Kim, Phys. Rev. Lett. 108 (2012) 177201.

[10] Y.-S. Chai, S. Kwon, S.-H. Chun, I. Kim, B.-G. Jeon, K.-H. Kim and S. Lee, Nature Communications 5 (2014) 4208.

[11] W. Eerenstein, M. Wiora, J. L. Prieto, J. F. Scott and N. D. Mathur, Nature Materials 6 (2007) 348.

[12] C. Thiele, K. Dörr, O. Bilani, J. Rödel and L. Schultz, Phys. Rev. B 75 (2007) 054408.

[13] S. Sahoo, S. Polisetty, C. G. Duan, S. S. Jaswal, E. Y. Tsymbal and C. Binek, Phys. Rev. B 76 (2007) 092108.

[14] S. Geprägs, A. Brandlmaier, M. Opel, R. Gross and S. T. B. Goennenwein, Appl. Phys. Lett. 96 (2010) 142509.

[15] T. Wu, A. Bur, P. Zhao, K. P. Mohanchandra, K. Wong, K. L. Wang, C. S. Lynch and G. P. Carman, Appl. Phys. Lett. 98 (2011) 012504.

[16] T. Wu, A. Bur, K. Wong, P. Zhao, C. S. Lynch, P. Khalili Amiri, K. L. Wang and G. P. Carman, Appl. Phys. Lett. 98 (2011) 262504.

[17] T. H. E. Lahtinen, K. J. A. Franke and S. van Dijken, Sci. Rep. 2 (2012) 258.

[18] S. Zhang, Y. G. Zhao, P. S. Li, J. J. Yang, S. Rizwan, J. X. Zhang, J. Seidel, T. L.Qu, Y. J. Yang, Z. L. Luo, Q. He, T. Zou, Q. P. Chen, J.W. Wang, L. F. Yang, Y. Sun, Y. Z. Wu, X. Xiao, X. F. Jin, J. Huang, C. Gao, X. F. Han and R. Ramesh, Phys. Rev. Lett. 108 (2012) 137203. 
[19] M. Buzzi, R.V. Chopdekar, J. L. Hockel, A. Bur, T. Wu, N. Pilet, P. Warnicke, G. P. Carman, L. J. Heyderman and F. Nolting, Phys. Rev. Lett. 111 (2013) 027204.

[20] R. O. Cherifi, V. Ivanovskaya, L. C. Phillips, A. Zobelli, I. C. Infante, E. Jacquet, V. Garcia, S. Fusil, P. R. Briddon, N. Guiblin, A. Mougin, A. A. Ünal, F. Kronast, S. Valencia, B. Dkhil, A. Barthélémy and M. Bibes, Nature Materials 13 (2014) 345.

[21] M. Ghidini, F. Maccherozzi, X. Moya, L .C. Phillips, W. Yan, J. Soussi, N. Métallier, M. E. Vickers, N. -J. Steinke, R. Mansell, C. H. W. Barnes, S. S. Dhesi and N. D. Mathur, Adv. Mater. 27 (2015) 1460.

[22] R. V. Chopdekar, M. Buzzi, C. Jenkins, E. Arenholz, F. Nolting and Y. Takamura, Sci. Rep. 6 (2016) 27501.

[23] Ya Gao, J.-M. Hu, C. T. Nelson, T. N. Yang, Y. Shen, L. Q. Chen, R. Ramesh and C. W. Nan, Sci. Rep. 6 (2016) 23696.

[24] P. Li, Y. Zhao, S. Zhang, A. Chen, D. Li, J. Ma, Y. Liu, D. T. Pierce, J. Unguris, H.-G. Piao, H. Zhang, M. Zhu, X. Zhang, X. Han, M. Pan and C.-W. Nan, ACS Appl. Mater. Interfaces 9 (2017) 2642.

[25] R. Lo Conte, Z. Xiao, C. Chen, C. V. Stan, J. Gorchon, A. El-Ghazaly, M. E. Nowakowski, H. Sohn, A. Pattabi, A. Scholl, N. Tamura, A. Sepulveda, G. P. Carman, R. N. Candler and J. Bokor, Nano Lett. 18 (2018) 1952.

[26] H. J. A. Molegraaf, J. Hoffman, C. A. F. Vaz, S. Gariglio, D. van der Marel, C. H. Ahn and J.M. Triscone, Adv. Mater. 21 (2009) 1.

[27] V. Skumryev, V. Laukhin, I. Fina, X. Martí, F. Sánchez, M. Gospodinov and J. Fontcuberta, Phys. Rev. Lett. 106 (2011) 057206.

[28] J. T. Heron, J. L. Bosse,Q. He,Y. Gao, M. Trassin, L. Ye, J. D. Clarkson, C. Wang, Jian Liu, S. Salahuddin, D. C. Ralph, D. G. Schlom, J. Íñiguez, B. D. Huey and R. Ramesh, Nature 516 (2014) 370.

[29] W. Saenrang, B. A. Davidson, F. Maccherozzi, J. P. Podkaminer, J. Irwin, R. D. Johnson, J. W. Freeland, J. Íñiguez, J. L. Schad, K. Reierson, J. C. Frederick, C. A. F. Vaz, L. Howald, T. H. Kim, S. Ryu, M. v. Veenendaal, P. G. Radaelli, S. S. Dhesi, M. S. Rzchowski and C. B. Eom, Nature Communications 8 (2017) 1583.

[30] V. Skumryev, V. Laukhin, I. Fina, X. Martí, F. Sánchez, M. Gospodinov and J. Fontcuberta, Phys. Rev. Lett. 106 (2011) 057206.

[31] N. Lei, T. Devolder, G. Agnus, P. Aubert, L. Daniel, J.-V. Kim, W. Zhao, T. Trypiniotis, R. P. Cowburn, C. Chappert, D. Ravelosona and Philippe Lecoeur, Nature Communications 4 (2013) 1378. 
[32] R. P. Cowburn, D. K. Koltsov, A. O. Adeyeye, M. E. Welland and D. M. Tricker, Phys. Rev. Lett. 83 (1999) 1042.

[33] T. Shinjo, T. Okuno, R. Hassdorf, K. Shigeto and T. Ono, Science 289 (2000) 930.

[34] A. Wachowiak, J. Wiebe, M. Bode, O. Pietzsch, M. Morgenstern and R. Wiesendanger, Science 298 (2002) 577.

[35] T. Wren, B. Gribkov, V. Petrashov and O. Kazakova1, J. Appl. Phys. 118 (2015) 023906.

[36] K. Watanabe, B. Jinnai, S. Fukami, H. Sato and H. Ohno, Nature Communications 9 (2018) 663.

[37] S. Bohlens, B. Krüger, A. Drews, M. Bolte, G. Meier and D. Pfannkuche, Appl. Phys. Lett. 93 (2008) 142508.

[38] K. Nakano, D. Chiba, N. Ohshima, S. Kasai, T. Sato, Y. Nakatani, K. Sekiguchi, K. Kobayashi and T. Ono, Appl. Phys. Lett. 99 (2011) 262505.

[39] B. Van Waeyenberge, A. Puzic, H. Stoll, K. W. Chou, T. Tyliszczak, R. Hertel, M. Fähnle, H. Brückl, K. Rott, G. Reiss, I. Neudecker, D. Weiss, C. H. Back and G. Schütz., Nature 444 (2006) 461.

[40] K. Yamada, S. Kasai, Y. Nakatani, K. Kobayashi, H. Kohno, A. Thiaville and T. Ono, Nature Materials 6 (2007) 270.

[41] S. Y. H. Lua, S. S. Kushvaha, Y. H. Wu, K. L. Teo and T. C. Chong, Appl. Phys. Lett. 93 (2008) 122504.

[42] M. Schneider, H. Hoffmann and J. Zweck, Appl. Phys. Lett. 79 (2001) 3113.

[43] V. Uhlír, M. Urbánek, L. Hladík, J. Spousta, M-Y. Im, P. Fischer, N. Eibagi, J. J. Kan, E. E. Fullerton and T. Šikola, Nature Nanotechnology 8 (2013) 341.

[44] Q. Li, A. Tan, A. Scholl, A. T. Young, M. Yang, C. Hwang, A. T. N'Diaye, E. Arenholz, J. Li and Z. Q. Qiu, Appl. Phys. Lett. 110 (2017) 262405.

[45] M. Ghidini, B. Zhu, R. Mansell, R. Pellicelli, A. Lesaine, X. Moya, S. Crossley, B. Nair, F. Maccherozzi, C. H. W. Barnes, R. P. Cowburn, S. S. Dhesi and N. D. Mathur, J. Phys. D: Appl. Phys. 51 (2018) 224007.

[46] A. Vansteenkiste, J. Leliaert, M. Dvornik, M. Helsen, F. Garcia-Sanchez and B. Van Waeyenberge, AIP Advances 4 (2014) 107133.

[47] A. Michels, J. Weissmüller, A. Wiedenmann and J. G. Barker J. Appl. Phys. 87 (2000) 5953.

[48] H. F. Kay and P. Vousden, Philos. Mag. 7 (1949) 1019. 